Received: 2018.11.22

Accepted: 2018.12.13

Published: 2019.04 .21
Authors' Contribution: Study Design A Data Collection B Statistical Analysis C Data Interpretation D Manuscript Preparation E Literature Search F Funds Collection G

\title{
Antiproliferative Effects of Matricine in Gemcitabine-Resistant Human Pancreatic Carcinoma Cells Are Mediated via Mitochondrial- Mediated Apoptosis, Inhibition of Cell Migration, Invasion Suppression, and Mammalian Target of Rapamycin (mTOR)-TOR/PI3K/AKT Signalling Pathway
}

$$
\begin{array}{rll}
\text { CEF 1 } & \text { Kaifeng Fang } \\
\text { BF 1 } & \text { Li Wang } \\
\text { CEF 1 } & \text { LuJia Chen } \\
\text { A } 2 & \text { Tao Liu }
\end{array}
$$

ADEG 1 Zhi Fang
1 Department of Emergency Surgery, Union Hospital, Tongii Medical College, Huazhong University of Science and Technology, Wuhan, Hubei, P.R. China 2 Department of Digestive Tumor Surgery, Union Hospital, Tongji Medical College, Huazhong University of Science and Technology, Wuhan, Hubei, P.R. China
Corresponding Author: Source of support:
Zhi Fang, e-mail: AlanxThomasbm@yahoo.com Departmental sources

Background: Pancreatic cancer is a major cause of mortality worldwide. Inefficient drugs, their adverse effects, and the development of drug resistance make it difficult to curb the growing incidence of pancreatic cancer. Against this backdrop, the development new drug regimens with no or negligible adverse effects is imperative. We assessed the anticancer effects of a plant-derived sesquiterpene - matricine - against capan-2 pancreatic cancer cells.

Material/Methods: Cell viability was determined by MTT assay. AO/EB, DAPI, and annexin V/PI staining were used to detect apoptosis. Transwell assays were used for monitoring of cell migration and invasion. Immunoblotting was used to examine the expression of proteins.

Results: The results showed that matricine halted the proliferation of capan-2 cells, with minimal toxic effects on normal pancreatic cells. The anticancer effects were due to the induction of apoptotic cell death, which was allied with activation of caspases 3 and 9, upregulation of Bax, and downregulation of Bcl-2. Moreover, matricine suppressed the migration and invasive abilities of pancreatic cancer cells at IC50. We also assessed the effects of matricine on the mTOR/PI3K/AKT signalling pathway. We found that matricine efficiently blocked this pathway, suggesting the anticancer potential of matricine.

Conclusions: Matricine induced antiproliferative effects in capan-2 human pancreatic cancer cells through inducing apoptosis, caspase activation, inhibition of cell migration and invasion, and blocking the mTOR/PI3K/AKT signalling pathway.

MeSH Keywords: Apoptosis • Cell Proliferation • Neoplasm Invasiveness • Pancreatic Neoplasms

Full-text PDF: $\quad$ https://www.medscimonit.com/abstract/index/idArt/914244

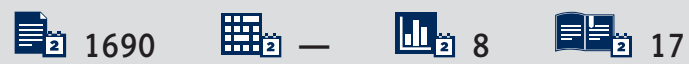




\section{Background}

Natural products have played an important role in curbing the incidence of cancer throughout the world. About half of the currently known anticancer drugs are derived from natural resources [1]. Drugs such as podophyllotoxin and Taxol are natural products and are currently used in the treatment of devastating cancers. Most anticancer drugs of natural origin come from plants [2]. Plants are always exposed to extremes of the environmental stresses and can't walk away from them. In response to the environmental stress and have evolved to cope with extreme conditions by synthesizing a wide diversity of chemical scaffolds, often referred to as secondary metabolites [3]. Although plant extracts were initially directly used for the treatment of ailments, with the advances in science and technology, the pure compounds isolated from plants are being used in the treatment of diseases as deadly as cancer [4]. Based on the structure of these chemical entities, they have been classified into different groups. Sesquiterpene is one such group of metabolites that is ubiquitously found across the plant kingdom and have been shown to exhibit promising bioactivities [5]. Sesquiterpenes from several plants have shown the capacity to inhibit the proliferation of cancers such as lung cancer, breast cancer, and gastric cancer [6].

In the present study we assessed the effects of matricine, a naturally occurring sesquiterpene [7] of plant origin, against gemcitabine-resistant Capan-2 human pancreatic carcinoma cells and non-cancerous hTRET-HPNE pancreatic cells. Pancreatic cancer is one of the deadliest cancers. In China and the United States, it is ranked as the seventh and fourth major causes of cancer related deaths, respectively [8]. It has been reported that pancreatic cancer affects 2.5 million people in the United States alone [9]. The prognosis of pancreatic cancer is the worst among all cancers [10]. Chemotherapy of pancreatic cancer has a number of adverse effects, and pancreatic cancer cells easily develop drug resistance, making it even more difficult to manage [11]. Moreover, pancreatic cancer frequently relapses and may also become drug resistant, making it difficult to manage [11]. Herein, we for the first time report the anticancer effects of matricine against capan-2 gemcitabine-resistant human pancreatic carcinoma cells. The results showed that matricine inhibits the growth of pancreatic cancer via induction of mitochondrial apoptosis and suppresses cell migration and invasion. Therefore, matricine may prove beneficial in the development of chemotherapy for pancreatic cancer.erH

\section{Material and Methods}

\section{Cell culture conditions}

The pancreatic cancer cell line Capan-2, and HeLa, the normal cell line hTRET-HPNE, were obtained from the Cancer Research
Institute of Beijing (Beijing, China) and maintained in Dulbecco's modified Eagle's medium (Invitrogen Life Technologies, MA) supplemented with $10 \%$ fetal bovine serum (Invitrogen Life Technologies, MA), 100 mg/ml streptomycin, and $100 \mathrm{U} / \mathrm{ml}$ penicillin G (Himedia, $\mathrm{PA}$ ) in an incubator at $37^{\circ} \mathrm{C}$ with $5 \% \mathrm{CO}_{2}$.

\section{Cell viability assay}

Briefly, at around 70\% confluence, the Capan-2 and the hTRETHPNE cells were seeded in 96-well plates and treated with $0-640 \mu \mathrm{M}$ of matricine. After incubation for $24 \mathrm{~h}$, the cells were incubated with MTT for $4 \mathrm{~h}$. After this, the medium was removed and the colored formazan product was solubilized by $200 \mu$ l of dimethyl sulfoxide. The viability of the capan-2 and the hTRET-HPNE cells was then determined by measuring absorbance at $570 \mathrm{~nm}$.

\section{DAPI (4',6-diamidino-2-phenylindole) and AO/EB (acridine orange/ethidium bromide) staining}

The Capan- 2 cells were grown in 6 -well plates $\left(0.6 \times 10^{6}\right.$ cells/well) and incubated for $12 \mathrm{~h}$. The Capan- 2 cells were subjected to matricine treatment at various levels $(0,10,20$, and $40 \mu \mathrm{M}$ ) for $24 \mathrm{~h}$ at $37^{\circ} \mathrm{C}$. As the cells sloughed off, $25 \mu \mathrm{l}$ cell cultures were put onto glass slides and stained with DAPI or a solution of $\mathrm{AO}$ and $\mathrm{EB}$. The slides were then covered with a cover slips and examined with a fluorescent microscope (BD Biosciences, San Jose, CA). The annexin V/PI staining was then performed as previously described [12].

\section{Cell migration and Invasion assay}

The migration and invasion abilities of Capan-2 cells were examined by Transwell chamber assay. In brief, $1 \times 10^{4}$ Capan- 2 cells were seeded in the upper chamber of the Transwell device (8- $\mu \mathrm{m}$ pore size polycarbonate filters) and treated with a $20-\mu \mathrm{M}$ concentration of matricine. This was followed by transfer into 24 -well plates and incubation at $37^{\circ} \mathrm{C}$ for $48 \mathrm{~h}$. For the invasion assay, the inserts were coated with extracellular matrix gel $(50 \mu \mathrm{l})(\mathrm{ECM}$, Sigma, USA). Swabbing was performed to remove the non-migrated and non-invaded cells from the upper surface, while the migrated and invaded cells on the lower surface were subjected to fixation with methanol for about $35 \mathrm{~min}$, followed by staining with crystal violet $(0.5 \%)$ for about $50 \mathrm{~min}$, then subjected to washing with PBS and finally counted under a light microscope ( 5 fields).

\section{Western blotting}

The Capan-2 cells were harvested and subjected to washing with ice-cold PBS. The pellets were then suspended in lysis buffer at $4^{\circ} \mathrm{C}$ and then incubated at $95^{\circ} \mathrm{C}$. Thereafter, the protein content of each cell extract was checked by Bradford assay. 


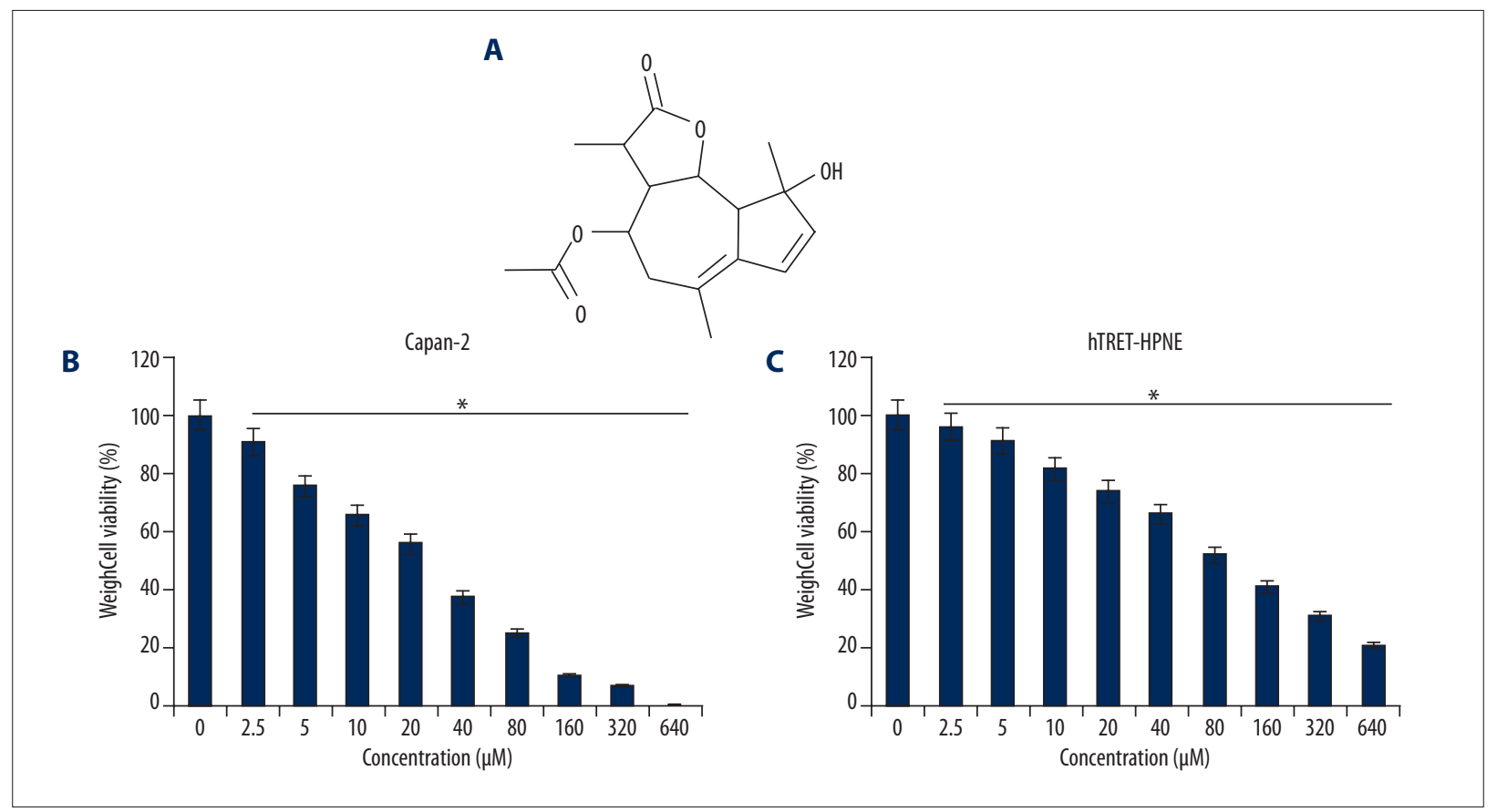

Figure 1. (A) Chemical structure of matricine. MTT assay showing the effect of matricine on the viability of (B) pancreatic capan-2 cells and (C) HTRET-HPNE non-cancerous cells. The experiments were performed in triplicate and results are shown as mean \pm SD $\left({ }^{*} P<0.05\right)$.

About $40 \mu \mathrm{g}$ of protein was loaded from each sample and separated by SDS-PAGE before being shifted to polyvinylidene fluoride membranes. The membranes were then subjected to treatment with TBS and exposed to primary antibodies at $4^{\circ} \mathrm{C}$. Thereafter, the cells were treated with appropriate secondary antibodies and the proteins of interest were visualized by enhanced chemiluminescence reagent.

\section{Statistical analysis}

The experiments were performed in triplicate and data are shown as mean \pm SD. Statistical analysis was done using the $t$ test with GraphPad prism 7 software. Values of $p<0.05$ were regarded as statistically significant differences.

\section{Results}

\section{Matricine inhibits the proliferation of pancreatic cancer cells}

The growth-inhibitory effects of matricine (Figure $1 \mathrm{~A}$ ) were examined on the capan-2 pancreatic cancer cells and the normal hTRET-HPNE cells by MTT assay at concentrations ranging from 0 to $640 \mu \mathrm{M}$. Matricine was found to halt the growth of capan-2 cells in a concentration-dependent manner (Figure $1 B$ ). The $I C_{50}$ of matricine against capan-2 cells was $20 \mu \mathrm{M}$. On the other hand, the effects of matricine on proliferation of TRET-HPNE cells were negligible. The $\mathrm{IC}_{50}$ of matricine against the normal hTRET-HPNE cells was $80 \mu \mathrm{M}$ (Figure $1 \mathrm{C}$ ).

\section{Matricine induces mitochondrial apoptotic cell death of pancreatic cancer cells}

Apoptosis in matricine-treated Capan-2 cells was determined by DAPI staining. It was quite evident from DAPI staining that the percentage of apoptotic cells increased with increase in the concentration of matricine (Figure 2). Moreover, AO/EB staining showed that the red fluorescent capan-2 cells increased upon treatment with matricine, indicative of apoptosis (Figure 3). The annexin V/PI staining of the matricine-treated cells showed that the apoptotic capan-2 cells increased from $1.2 \%$ in control to $48.4 \%$ at $40 \mu \mathrm{M}$ of matricine (Figure 4). The apoptosis of the matricine-treated capan-2 cells was further validated by examining the levels of apoptosis-related proteins by Western blot analysis, showing that Matricine activated caspase-3 and -9 expression in a concentration-dependent manner. Further, the expression of Bax was increased but expression of $\mathrm{Bcl}-2$ was decreased by matricine treatment (Figure 5).

\section{Matricine inhibits cell migration and invasion of pancreatic cancer cells}

Next, the effects of matricine on the migration and invasion of capan-2 cancer cells were investigated by Transwell assays. The results showed that at $I C_{50}$, matricine inhibited the 


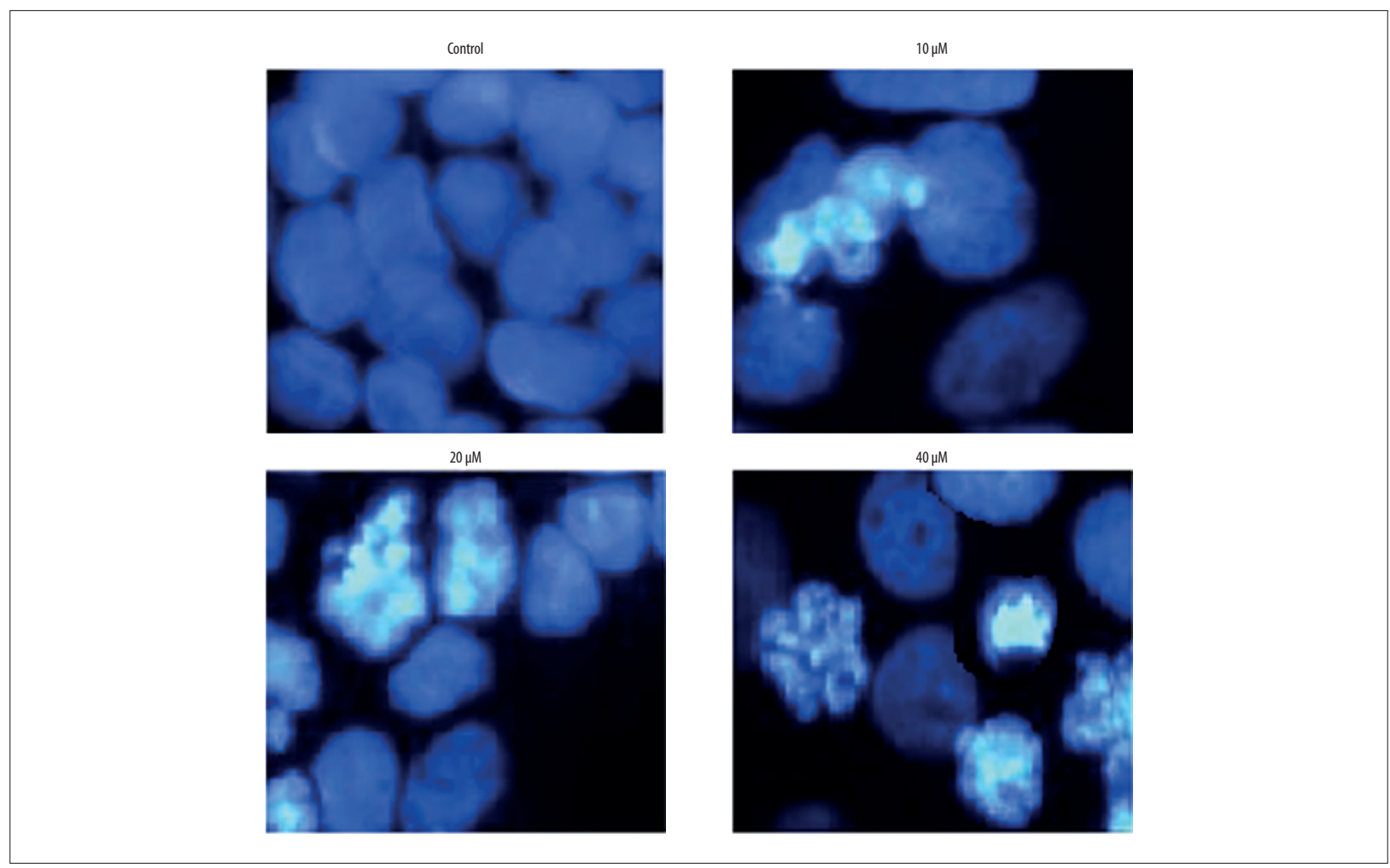

Figure 2. DAPI staining showing the apoptosis-inducing effect of matricine on capan-2 cells. The experiments were performed in triplicate. The figure shows that matricine induces apoptosis in capan-2 cells in a concentration-dependent manner.

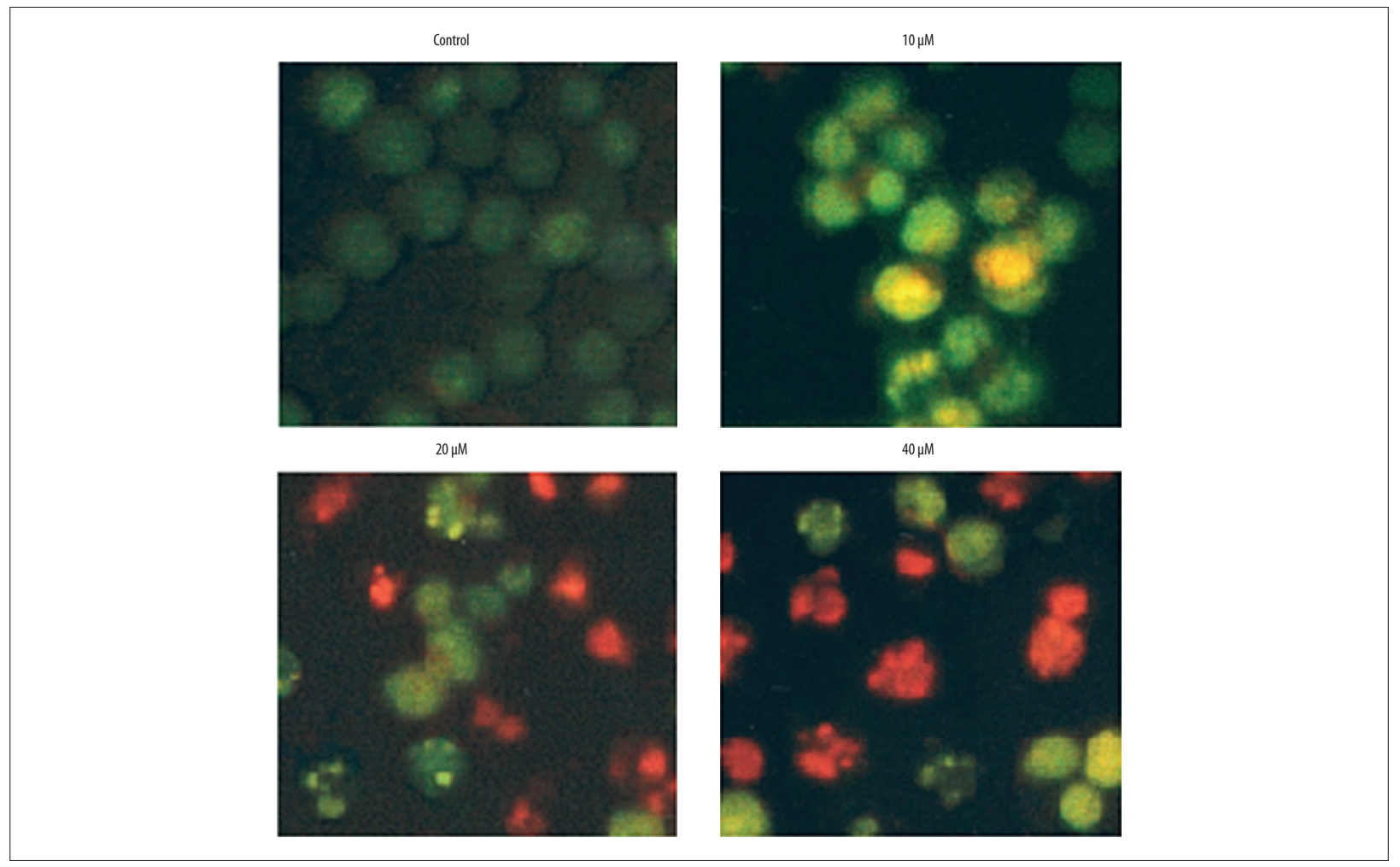

Figure 3. AO/EB staining showing the apoptosis-inducing effect of matricine on capan-2 cells. The experiments were performed in triplicate. The figure shows that matricine triggers apoptosis in capan-2 cells in a concentration-dependent manner. 


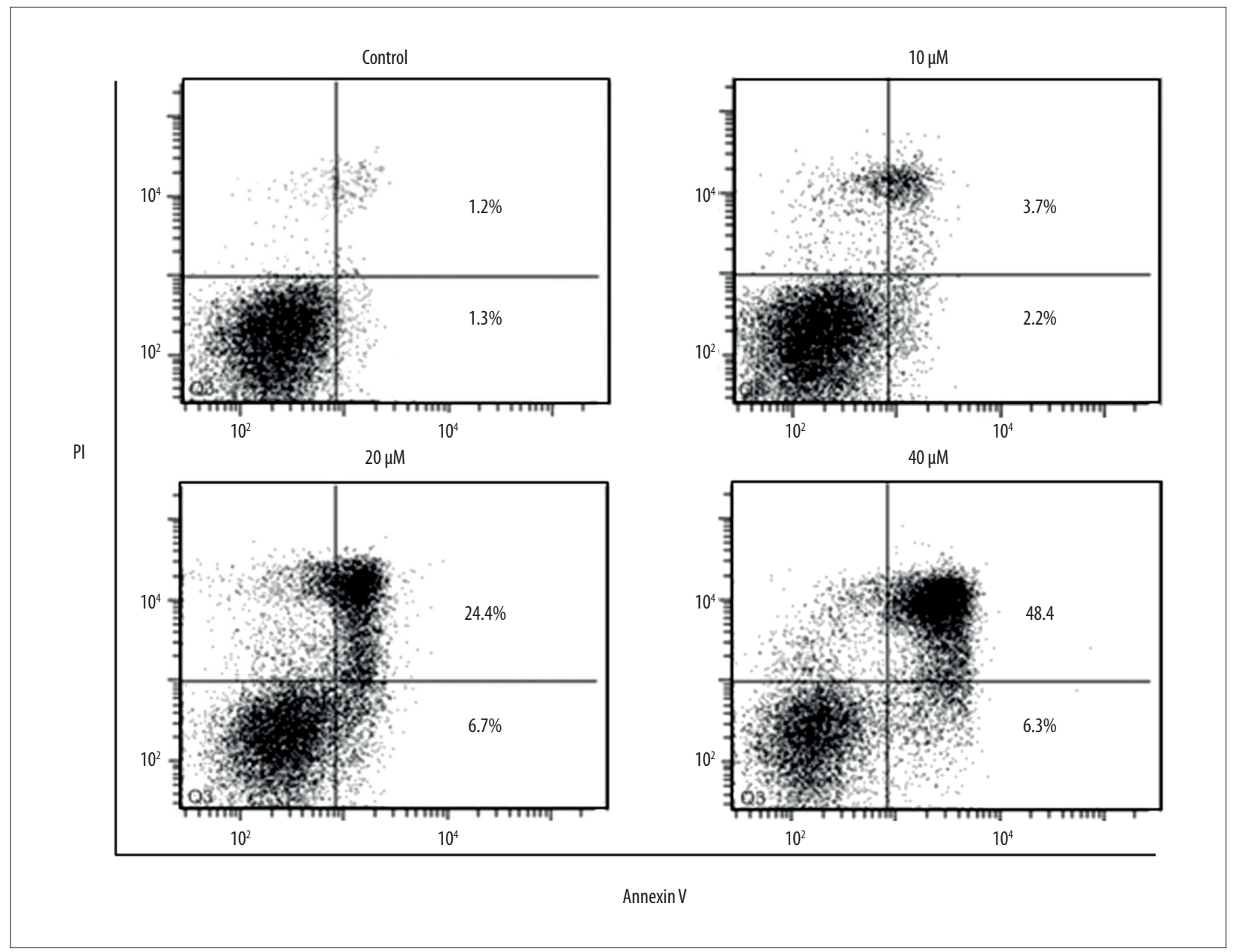

Figure 4. Annexin V/PI staining showing the percentage of apoptosis in matricine-treated capan-2 cells. The experiments were performed in triplicate. The figure shows that the apoptotic cell populations increased with increased concentration of matricine.

migration of capan-2 cancer cells (Figure 6). A similar trend was observed with cell invasion (Figure 7).

\section{Matricine inhibits the mTOR/PI3K/AKT signalling pathway}

Next, we assessed the effects of matricine on the mTOR/PI3K/ AKT signalling pathway of capan-2 pancreatic cancer cells. We found that matricine caused a significant decline in the expression of mTOR, PI3K, and AKT. These inhibitory effects of matricine exhibited a dose-dependent trend. Further, matricine also inhibited the phosphorylation of mTOR, PI3K, and AKT in a concentration-dependent manner (Figure 8).

\section{Discussion}

Pancreatic carcinoma is one of the most commonly diagnosed cancers throughout the world [13]. The adverse effects of treatment strategies and the unavailability of therapeutic targets

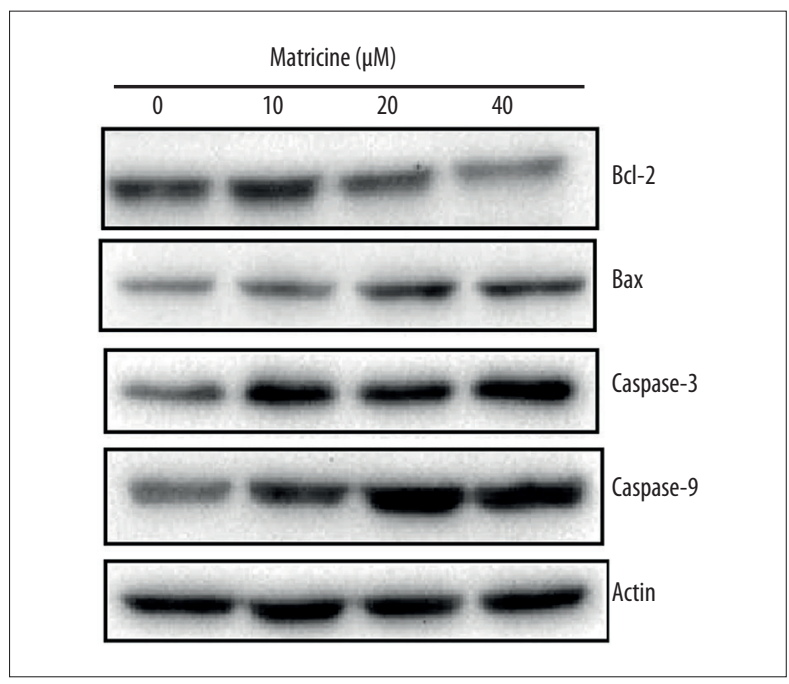

Figure 5. Effect of matricine on apoptosis-related protein expression at indicated concentrations. The experiments were performed in triplicate. 

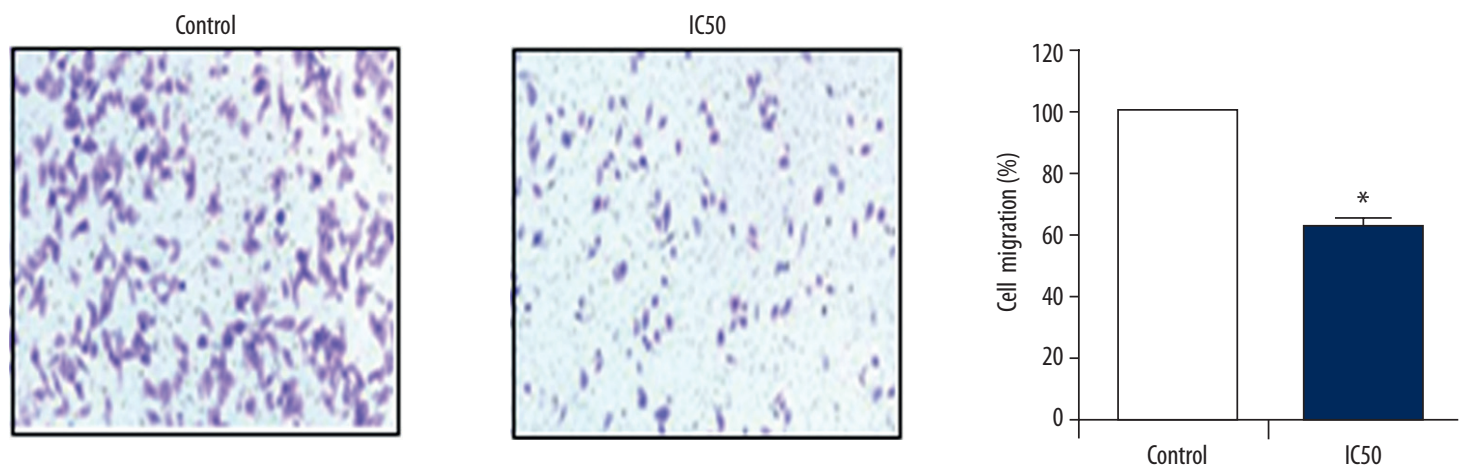

Figure 6. Effect of matricine on the migration of capan-2 cells. The experiments were performed in triplicate and results are shown as mean $\pm \mathrm{SD}\left({ }^{*} P<0.05\right)$.

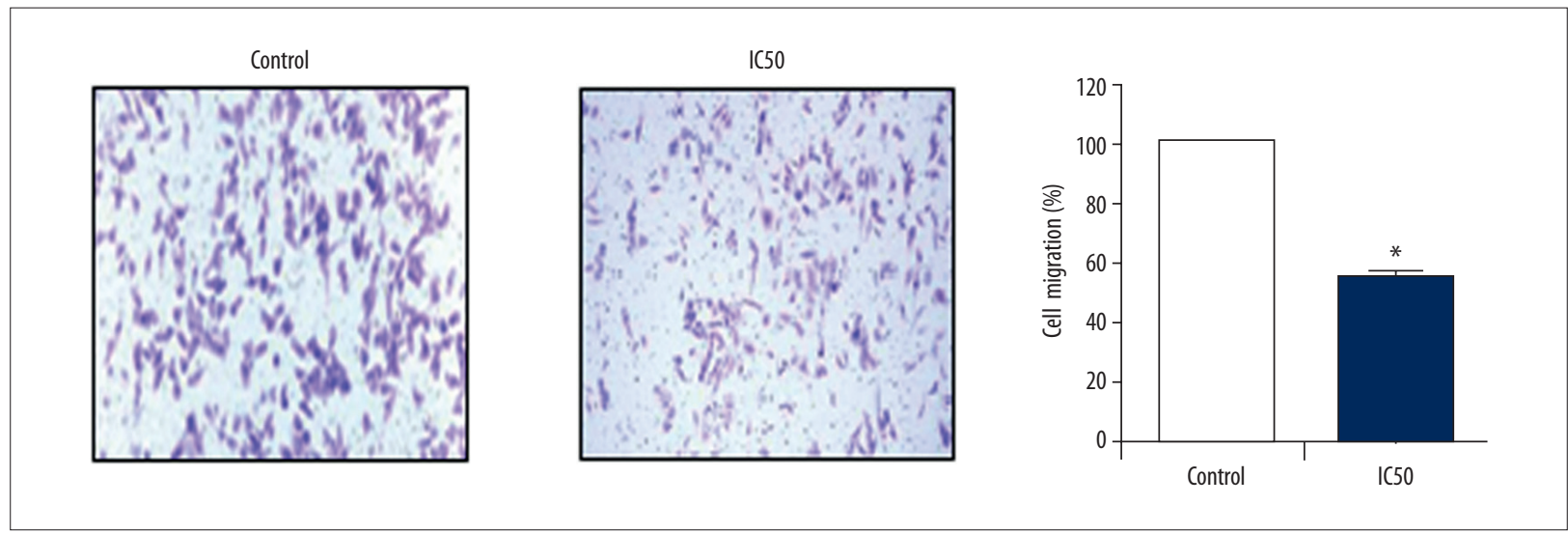

Figure 7. Effect of matricine on the invasion of capan-2 cells. The experiments were performed in triplicate and results are shown as mean $\pm \mathrm{SD}\left({ }^{*} P<0.05\right)$.

are the main obstacles that limit its treatment [10]. Moreover, the emergence of drug resistance in pancreatic cancer cells makes it very difficult to treat pancreatic cancer with existing drugs [10]. Plants are a diverse repository of chemical entities that may prove to be essential in the development of safer chemotherapy for the treatment of pancreatic cancer $[2,3]$. In the present study we assessed the anticancer effects of matricine, a plant-derived sesquiterpene, against capan-2 pancreatic cancer cells and normal pancreatic HTRET-HPNE cells. We found that matricine exerted dose-dependent growth effects on the capan-2 cells. However, matricine showed minimal toxic effects on normal HTRET-HPNE cells. These results are in agreement with investigations in which Sesquiterpenes from the clove plant were shown to suppress the growth of cancer cells [6]. Previous studies have shown that plant-derived anticancer molecules exert their anticancer effects via multiple mechanisms such as apoptosis, autophagy, and cell cycle arrest [14]. We performed DAPI and AO/EB staining of matricine capan-2 cells, showing that matricine treatment causes apoptotic cell death of Capan-2 pancreatic cancer cells. Annexin V/PI staining also showed that the apoptotic cell percentage

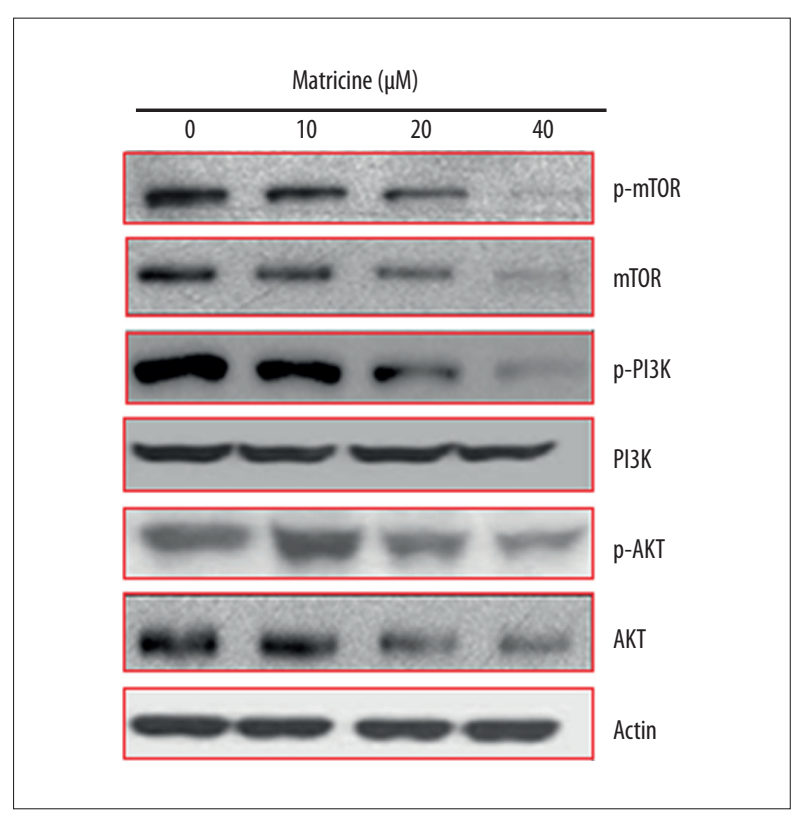

Figure 8. Effect of matricine on mTOR/PI3K/AKT signalling pathway at indicated concentrations of matricine. The experiments were performed in triplicate. 
increased with increased concentrations of matricine. Apoptosis is an essential process to eliminate cancer cells to maintain tissue homeostasis and is indicative of the potential of matricine as an anti-cancer agent [15]. Moreover, the matricine-induced apoptosis was also accompanied by activation of caspases 3 and 9, upregulation of Bax, and decreased levels of Bcl-2. Cell migration and invasion are pre-requisites for metastasis of cancer cells [16]. Herein, we investigated whether matricine has any apparent effect on migration and invasion of Capan-2 pancreatic cancer cells by Transwell assays. Interestingly, we found that matricine significantly suppressed the migration and invasion abilities of capan- 2 cells. The mTOR/PI3K/AKT signalling pathway is one of the essential therapeutic targets for anticancer drugs owing to the involvement of this pathway in the development, progression, and tumorigenesis of cancers [17]. We found that matricine efficiently blocks this pathway in cancer cells, indicative of the potential of matricine as an anticancer drug.

\section{References:}

1. Phillipson JD: Natural products as drugs. Trans R Soc Trop Med Hyg, 1994; 88(Suppl. 1): 17-19

2. Tietze LF, Modi A: Multicomponent domino reactions for the synthesis of biologically active natural products and drugs. Med Res Rev, 2000; 20(4): 304-22

3. Choi EJ, Lee J, Kim GH: Evaluation of the anticancer activities of thioflavanone and thioflavone in human breast cancer cell lines. Int J Mol Med, 2012; 29(2): 252-56

4. Han R: Highlight on the studies of anticancer drugs derived from plants in China. Stem Cells, 1994 ;12(1): 53-63

5. Moraes DF, de Mesquita LS, do Amaral FM et al: Anticancer drugs from plants. In: Biotechnology and production of anti-cancer compounds. Springer, Cham, 2017; 121-42

6. Ren Y, Yu J, Kinghorn AD: Development of anticancer agents from plant-derived sesquiterpene lactones. Curr Med Chem, 2016; 23: 2397-420

7. Ness A, Metzger JW, Schmidt PC: Isolation, identification and stability of 8-desacetylmatricine, a new degradation product of matricine. Pharmaceutica Acta Helvetiae, 1996; 71(4): 265-71

8. Jemal A, Siegel R, Xu J, Ward E: Cancer statistics. Cancer J Clin, 2010; 60: 277-300

9. Chen W, Zheng R, Zhang S et al: Report of incidence and mortality in China cancer registries, 2009. Chin J Cancer Res, 2013; 25: 10-21

\section{Conclusions}

Matricine inhibited the growth of pancreatic cancer with minimal toxicity to normal cells. Matricine triggered apoptosis and inhibited the migration and invasion abilities of pancreatic cancer cells via PI3K/AKT/mTOR cascade. Therefore, matricine shows potential in the development of new chemotherapy drugs, but more research on this topic is needed.

\section{Conflict of interest}

None.

10. Hirata K, Egawa S, Kimura $Y$ et al: Current status of surgery for pancreatic cancer. Dig Surg, 2007; 24: 137-47

11. Gillen S, Schuster T, Meyer C et al: Preoperative/neoadjuvant therapy in pancreatic cancer: A systematic review and meta-analysis of response and resection percentages. PLoS Med, 2010; 7, e1000267

12. Hua F, Li CH, Chen XG, Liu XP: Daidzein exerts anticancer activity towards SKOV3 human ovarian cancer cells by inducing apoptosis and cell cycle arrest, and inhibiting the Raf/MEK/ERK cascade. Int J Mol Med, 2018; 41(6): 3485-92

13. Zhang $Q$, Zeng L, Chen $Y$ et al: Pancreatic cancer epidemiology, detection, and management. Gastroenterol Res Pract, 2016; 2016: 8962321

14. Lowe SW, Lin AW: Apoptosis in cancer. Carcinogenesis, 2000; 21(3): 485-95

15. Ouyang L, Luo Y, Tian $M$ et al: Plant natural products: from traditional compounds to new emerging drugs in cancer therapy. Cell Prolif, 2014; 47(6): 506-15

16. Zhou H, Zhang B, Zheng J et al: The inhibition of migration and invasion of cancer cells by graphene via the impairment of mitochondrial respiration. Biomaterials, 2014; 35(5): 1597-607

17. Neri LM, Cani A, Martelli AM et al: Targeting the PI3K/Akt/mTOR signaling pathway in B-precursor acute lymphoblastic leukemia and its therapeutic potential. Leukemia, 2014; 28(4): 739-48 